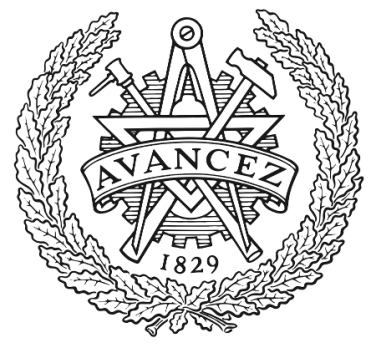

CHALMERS

UNIVERSITY OF TECHNOLOGY

\title{
Spatial Power Combining and Splitting in Gap Waveguide Technology
}

Downloaded from: https://research.chalmers.se, 2023-04-26 11:41 UTC

Citation for the original published paper (version of record):

Maaskant, R., Shah, W., Uz Zaman, A. et al (2016). Spatial Power Combining and Splitting in Gap

Waveguide Technology. IEEE Microwave and Wireless Components Letters, 26(7): 472-474.

http://dx.doi.org/10.1109/lmwc.2016.2574828

N.B. When citing this work, cite the original published paper.

C2016 IEEE. Personal use of this material is permitted.

However, permission to reprint/republish this material for advertising or promotional purposes 


\title{
Spatial Power Combining and Splitting in Gap Waveguide Technology
}

\author{
Rob Maaskant, Senior Member, IEEE, Waqar Ali Shah, Ashraf Uz Zaman, Member, IEEE, \\ Marianna Ivashina, Senior Member, IEEE, and P.-S. Kildal, Fellow, IEEE
}

\begin{abstract}
A single-layer spatial power splitter-combiner structure is packaged in gap waveguide technology. The measured metal-only back-to-back structure features an average insertion loss less than $2.3 \mathrm{~dB}$ and a return loss larger than 10 to $20 \mathrm{~dB}$ over the entire $75-110 \mathrm{GHz} \mathrm{W}$-band. The design procedure is outlined and the measured and simulated results are shown to be in good agreement. The structure can be used as a stand-alone power splitter and/or combiner (single-mode, all-excited in-phase channels), a quasi-optical beamformer to excite an array of slot antennas in the top ground plane by ridge gap waveguides, or as a back-to-back RF component in grid amplifier designs.
\end{abstract}

Index Terms-Gap waveguide technology, spatial power combining.

\section{INTRODUCTION}

$\mathbf{T}$ HE design of high-performance low-loss RF systems that are energy efficient, inexpensive and packaged in a single unit has gained significant attention, particularly for high-frequency applications where system integration becomes a necessity. However, several major challenges need to be overcome.

Firstly, insertion losses of feed networks and power combiners based on printed circuit technology suffer from both dielectric and conductor losses, which increase with frequency. These losses become even larger if the number of splitter (or combiner) channels increases, see e.g. [1, Fig. 1]. To some extent, dielectric-free transmission lines are capable to mitigate this problem [2]. Still, if currents are concentrated to narrow transmission lines, quadratically larger conductor losses are to be expected as compared to when currents are distributed over larger surfaces (on account of $I^{2} R$ ). Hence, spatial power combining techniques are preferred.

Secondly, RF components may radiate electromagnetic fields due to discontinuities (transitions, bends, interconnects) which will excite cavity resonances when the circuit is packaged. This, in turn, will lead to the problem of unwanted feedback effects between components causing packaged devices to oscillate, and so on.

The metamaterial-based gap waveguide (GW) concept is not only a low-loss dielectric-free transmission line structure, but it also represents a resonance-free packaging solution that is capable to overcome the above-mentioned problems [3],

The authors are with the Department of Signals and Systems (Division of Antenna Systems), Chalmers University of Technology, S-41296 Gothenburg, Sweden. e-mail: rob.maaskant@chalmers.se.

Manuscript received Nov. 16, 2015; revised Feb. 01, 2016. This work was supported by the European and Swedish Research Councils: an advanced investigator grant (no. 321125), a Swedish Young Researcher grant, and VINNOVA within the VINN Excellence Center Chase at Chalmers.
[4]. To date, spatial power combining techniques have not yet been realized in GW technology. This is an interesting research direction because it may lead to packaged grid amplifiers obviating the use of dielectric lenses and bulky horn antennas [1], as clarified in some more detail later.

This paper presents the design steps and measured results of a single layer wide bandwidth spatial power combiner and splitter in GW technology. The novel design forms the basis for several passive and active components to be designed in future. Among them a beamforming component for slot antenna arrays, see e.g. [5] combined with the spatial power combiner in the (more lossy) substrate integrated waveguide technology [6]. Furthemore, as a back-to-back (B2B) structure, it may be employed in the design of power grid amplifiers, or half of it as a stand-alone power splitter or combiner.

The paper is organized as follows. Sec. II presents the B2B structure; Sec. III the design steps; Sec. IV the measured and simulated results; and Sec. V contains the conclusions.

\section{The Proposed Back-to-Back Structure}

The proposed B2B structure is shown in Fig. 1. The field

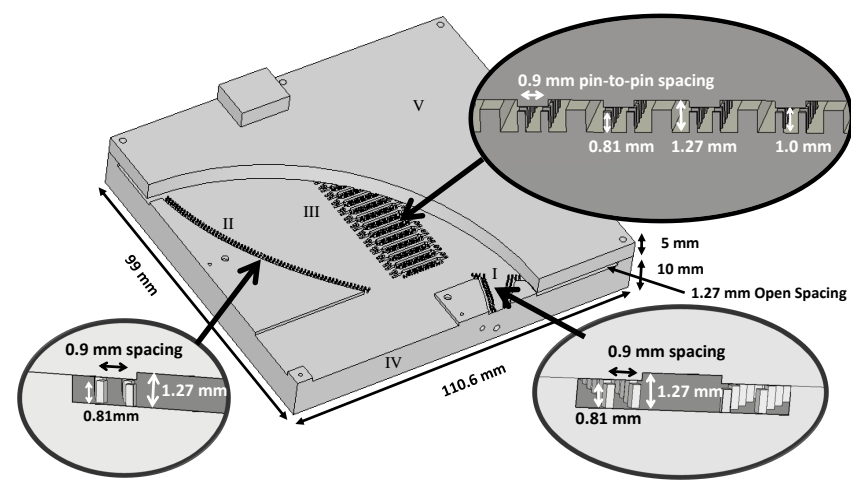

Fig. 1. The single layer B2B prototype. I: horn antenna; II: parabolicallyshaped reflecting pin wall groove GW); III: array of ridge GWs; IV and V: bottom and top block forming parallel-plate waveguide region, respectively.

of a standard WR-10 rectangular waveguide (W-band, 75-110 $\mathrm{GHz}$ ) enters the structure and excites the GW horn antenna I. The horn illuminates a wall of groove GW pins representing an offset parabolic reflector - similar to II but on the opposite side. The planar wavefront emanating from the reflector is then incident on an array of ridge GWs (III). The ridges are separated by GW pins creating a stop band - thus blocking all modes. This increases the ridge-to-ridge isolation $(\sim 20$ $\mathrm{dB}$ per pin row, $c f$. Fig. 6), as opposed to laterally leaking rectangular waveguides made of $\mathrm{H}$-plane split blocks. The 
field distributions in Fig. 2 further exemplify the operation principle.
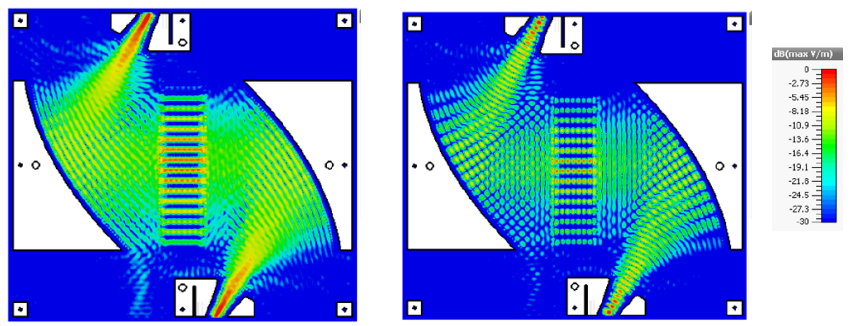

Fig. 2. (left) Time-averaged E-field amplitude, and; (right) instantaneous field @ $80 \mathrm{GHz}$ inside the parallel-plate waveguide structure.

The purpose of this letter is to describe the optimized design and specific features of the above B2B structure and to report on the prototype measurements.

\section{DESIGN STEPS}

As we aim for W-band operation, the first step is to design a bed of nails exhibiting a stop band for this frequency range [7]. Figure 3 shows the computed dispersion diagram of a single pin inside a unit cell employing periodic boundary conditions (CST software is used). No modes are seen to be present in

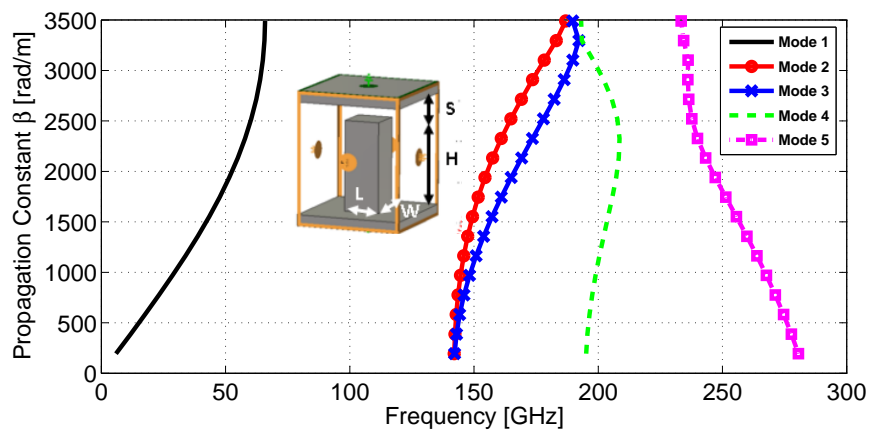

Fig. 3. Dispersion diagram of a GW pin showing the stop band.

the band from 70-140 GHz. The GW pin dimensions are $L=$ $W=0.3 \mathrm{~mm}, H=0.81 \mathrm{~mm}$, and $S=0.19 \mathrm{~mm}$. The pin period is $0.9 \mathrm{~mm}$.

The next step was to design the horn antenna and the offset parabolic reflecting wall in groove GW technology. The optimized design of a single feed-reflector transition is shown in Fig. 4. The reflector projected aperture size is fixed at $13.8 \lambda$ at the lowest frequency of operation $(75 \mathrm{GHz})$. The reflector semi-subtended angle $\Theta_{\mathrm{s}} / 2=36.3$ degrees. The feed horn opening of $2 \lambda$ has been determined through a receive-mode analysis, viz. by letting a tapered plane wave be incident on the reflector wall and by making sure that the horn intercepts more than $95 \%$ of the total incident power. The waveguide bend needed optimization to minimize the input reflection loss. The horn feed has an aperture pin-extension up to $4 \lambda$ to minimize spillover loss (reflector edge illumination $<-30 \mathrm{~dB}$ ). The simulated input reflection coefficient is shown in Fig. 5. It is evident that the horn feed is extremely well matched, i.e., $\left|S_{11}\right|<-25 \mathrm{~dB}$, and that the presence of the reflector induces only minor ripples on the input reflection coefficient.
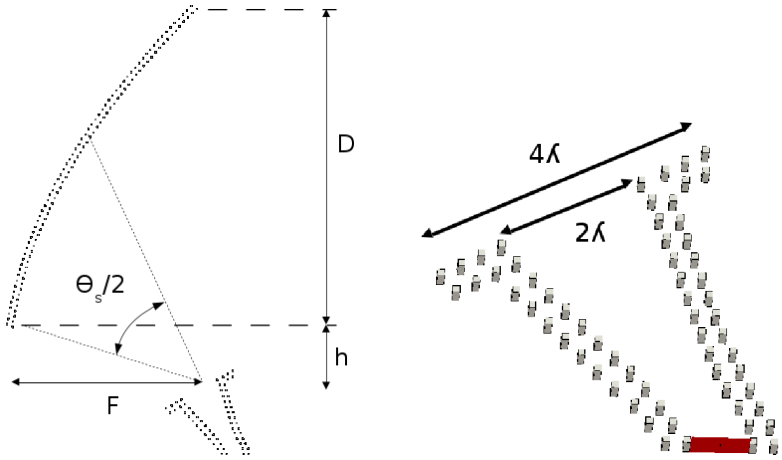

Fig. 4. (left) Feed-reflector design parameters (in mm): $D=55 ; h=10$, and; $F=32.5$. Hence, $F / D=0.59$. (right) close-up of the $\mathrm{GW}$ horn.

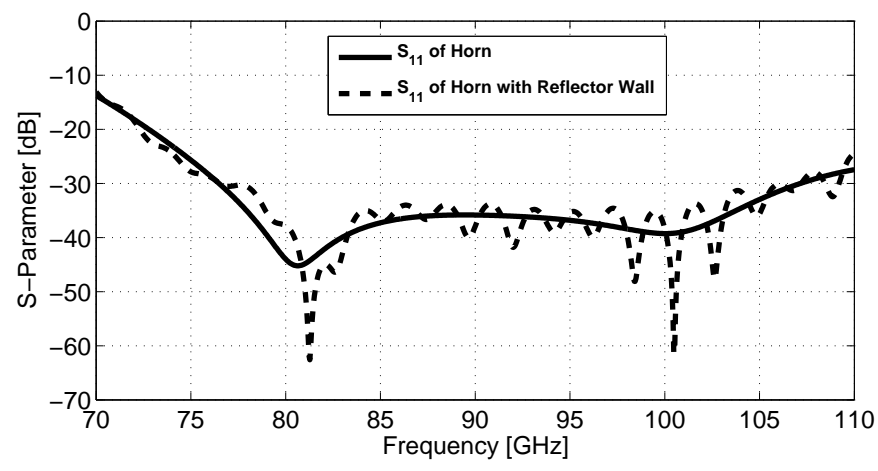

Fig. 5. Horn feed input reflection coefficient with and without reflecting wall.

The next step is to design the transition from planar wavefront of the parallel-plate waveguide field emanating from the reflector aperture to an array of ridge GWs, i.e., the center section in Figs. 1 and 2. Fig. 6 is a close-up of the designed transition. The ridges are isolated by GW pins and end in a fork-type structure on both sides to minimize reflection losses. If only a single stepped ridge transformer is used, the incident fields would be disrupted too much by the pins that isolate the ridges. The forks are tailored to 'pick-up' the field before it reaches the pins. The array of ridge GWs has

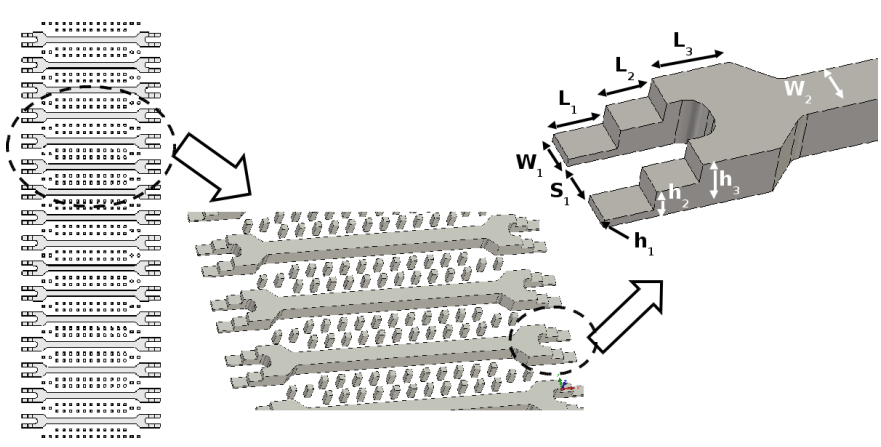

Fig. 6. The fork structure as a transition from parallel-plate waveguide fields to an array of ridge GWs.

been optimized by taking two oversized waveguide ports on either side of the structure in Fig. 6 (left) and excite it from one side by an incident $T E_{10}$ waveguide mode, while matchterminate all higher-order scattered modes traveling back into both ports. The incident field is a good representation of the 
tapered field that emanates from the reflector aperture. The level of tapering depends on the feed-reflector design. If the ridges were to excite slot antennas in the top ground plane, amplitude tapering is desired to reduce the side-lobe level, whereas uniform amplitudes and phases across the ridges are desired in case of grid amplifier designs.

The simulated time-averaged amplitude of the field distribution (E-field) is shown in Fig. 7(a). The simulated S-
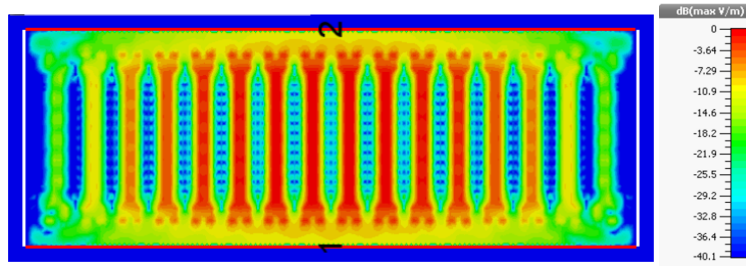

(a)

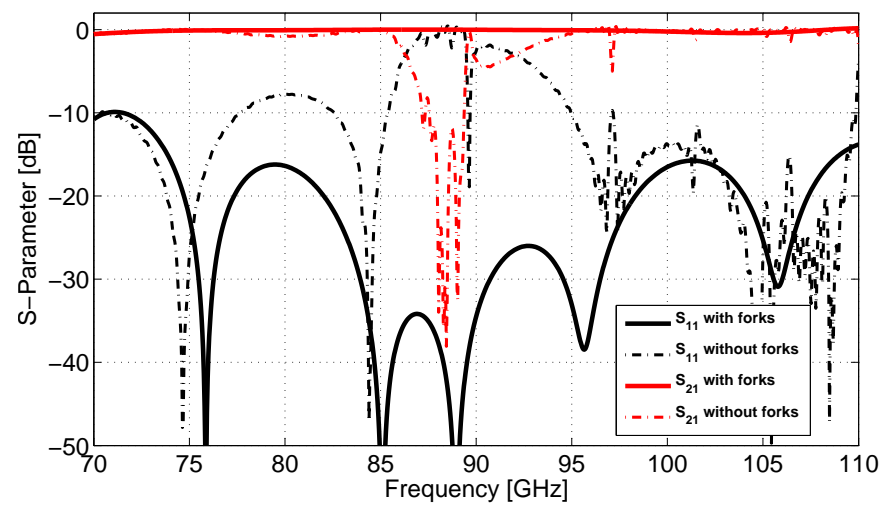

(b)

Fig. 7. (a) Time-average E-field amplitude of the fork structure. (b) Sparameters with and without fork structure.

parameters are shown in Fig. 7(b), with and without fork structure. The $S_{11}$ for the fork structure is below $-15 \mathrm{~dB}$ over almost the entire W-band, whereas the performance is severely compromised for single tapered ridges. The final dimensions of the optimized forks are (in mm): $L_{1}=0.7821 ; L_{2}=0.68$; $L_{3}=1.25 ; h_{1}=0.15 ; h_{2}=0.52 ; h_{3}=0.89 ; W_{1}=0.537 ;$ $W_{2}=0.774$, and; $S_{1}=0.774$.

\section{Measurement Results}

After optimizing each component of the B2B structure, an aluminum prototype was milled with 5-10 $\mu \mathrm{m}$ acceptable tolerance and then measured by a VNA using standard WR-10 rectangular waveguide flanges and extension modules, as shown in Fig. 8. The simulated and measured S-parameter of the entire strucutre are shown in Fig. 9. The simulated $S_{11}$ is less than $-12 \mathrm{~dB}$ over the the entire W-band, while the average insertion loss is only about $0.65 \mathrm{~dB}$ for PEC materials. Note the open slits on the sides as the top metal plate only needs spacers to support it. The measured $S_{11}$ remains below $-10 \mathrm{~dB}$ over the entire W-band, but the $S_{21}$ degraded to $-2.28 \mathrm{~dB}$. This is partly attributed to the surface roughness, as also the simulation indicates in Fig. 9, where the $S_{21}$ is seen to degrade from $-0.65 \mathrm{~dB}$ (PEC materials) to $-1.67 \mathrm{~dB}$ (for $15 \mu \mathrm{m}$ surface roughness).
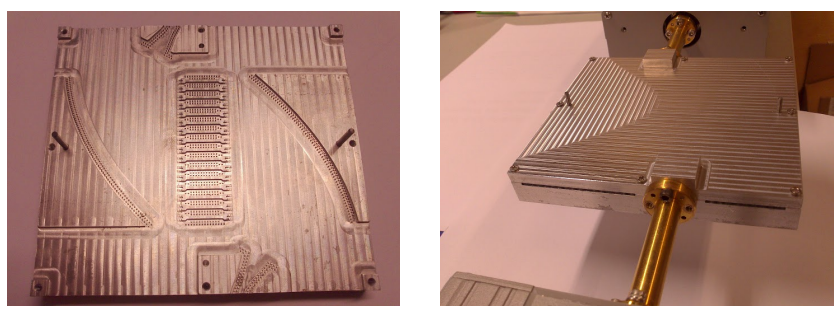

Fig. 8. (left) Interior of the milled prototype. (right) VNA measurement setup.

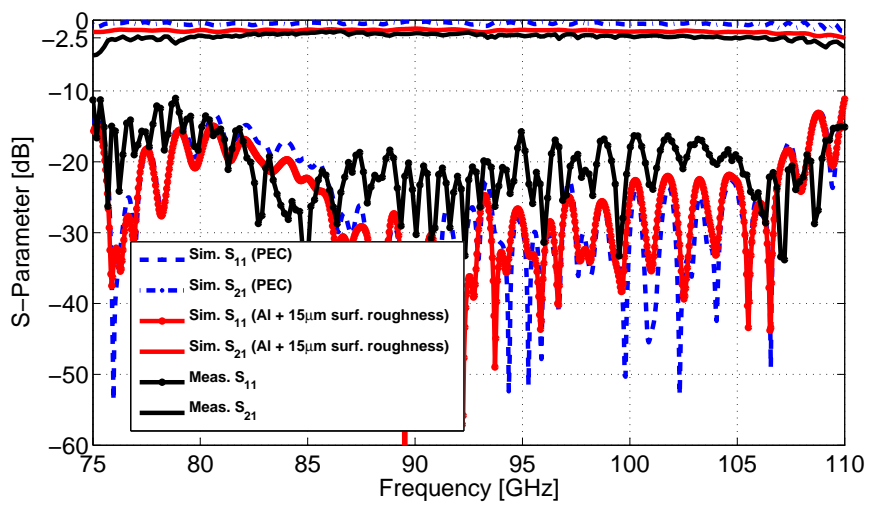

Fig. 9. Measured and simulated S-parameter results for the B2B structure.

\section{CONCLUSIONS}

The 16-channel back-to-back gap waveguide spatial power splitter/combiner has an $S_{11}<-10 \mathrm{~dB}$ and an $S_{21}>$ $-2.28 \mathrm{~dB}$ over the entire $\mathrm{W}$-band $(1.1 \mathrm{~dB}$ half the structure, no surface treatment). A fork-type transition is needed to transfer the quasi-optically beamformed parallel-plate waveguide field to the array of ridge gap waveguides. The novel dielectric-free structure can be used as a stand-alone power splitter and/or combiner, a beamformer to excite an array of slot antennas in the top ground plane by ridge gap waveguides, or as a back-toback structure for the design of planar grid amplifiers. Future work will be focusing toward these applications.

\section{REFERENCES}

[1] M. P. DeLisio and R. A. York, "Quasi-optical and spatial power combining," IEEE Trans. Microw. Theory Tech., vol. 50, no. 3, pp. 929-936, Mar. 2002.

[2] A. U. Zaman and P.-S. Kildal, "Wide-band slot antenna arrays with singlelayer corporate-feed network in ridge gap waveguide technology," IEEE Trans. Antennas Propag., vol. 62, no. 6, pp. 2992-3001, Jun. 2014.

[3] P.-S. Kildal, E. Alfonso, A. Valero-Nogueira, and E. Rajo-Iglesias, "Local metamaterial-based waveguides in gaps between parallel metal plates," IEEE Antennas Wireless Propag. Lett., vol. 8, no. 1, pp. 84-87, 2009.

[4] A. Valero-Nogueira, E. Alfonso, J. I. Herranz, and P.-S. Kildal, "Experimental demonstration of local quasi-TEM gap modes in single hard-wall waveguides," IEEE Microw. Wireless Compon. Lett., vol. 19, pp. 536-538, 2009.

[5] M. A. Sharkawy and A. Kishk, "Wideband beam scanning circularly polarized inclined slots using ridge gap waveguide," IEEE Antennas Wireless Propag. Lett., vol. 13, pp. 1187-1190, Jun. 2014.

[6] E. Gandini, M. Ettorre, M. Caseletti, K. Tekkouk, L. L. Coq, and R. Sauleau, "SIW slotted waveguide array with pillbox transition for mechanical beam scanning," IEEE Antennas Wireless Propag. Lett., vol. 11, pp. 1572-1575, 2012.

[7] E. Rajo-Iglesias and P.-S. Kildal, "Numerical studies of bandwidth of parallel-plate cut-off realised by a bed of nails, corrugations and mushroom-type electromagnetic bandgap for use in gap waveguides," IET Microwaves, Antennas \& Propagation, vol. 5, no. 3, pp. 282-289, 2011. 